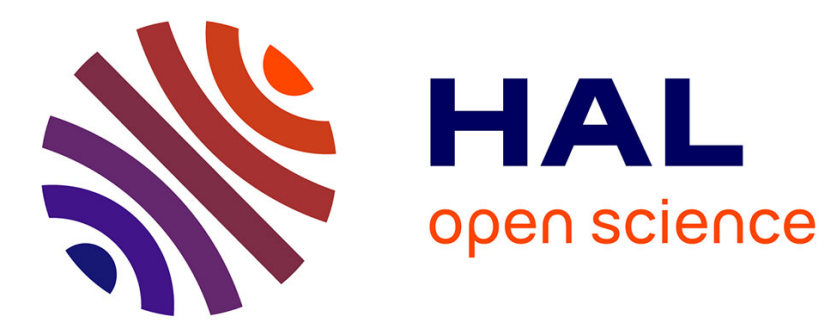

\title{
A Single-Molecule Digital Full Adder
}

We -Hyo Soe, Paula de Mendoza, Antonio M Echavarren, Christian Joachim

\section{To cite this version:}

We -Hyo Soe, Paula de Mendoza, Antonio M Echavarren, Christian Joachim. A SingleMolecule Digital Full Adder. Journal of Physical Chemistry Letters, 2021, 12 (35), pp.8528-8532. 10.1021/acs.jpclett.1c02449 . hal-03412802

\section{HAL Id: hal-03412802 https://hal.science/hal-03412802}

Submitted on 3 Nov 2021

HAL is a multi-disciplinary open access archive for the deposit and dissemination of scientific research documents, whether they are published or not. The documents may come from teaching and research institutions in France or abroad, or from public or private research centers.
L'archive ouverte pluridisciplinaire HAL, est destinée au dépôt et à la diffusion de documents scientifiques de niveau recherche, publiés ou non, émanant des établissements d'enseignement et de recherche français ou étrangers, des laboratoires publics ou privés. 


\section{A Single Molecule Digital Full Adder}

We-Hyo Soe, ${ }^{*+}$ Paula de Mendoza, ${ }^{\S}$ Antonio M. Echavarren ${ }^{\S}$ and Christian Joachim ${ }^{\dagger t}$

${ }^{\dagger}$ Centre d'Elaboration de Matériaux et d'Études Structurales (CEMES), Centre National de la Recherche Scientifique (CNRS), Université de Toulouse, 29 Rue J. Marvig, BP 94347, 31055 Toulouse Cedex, France

$\ddagger$ International Center for Materials Nanoarchitectonics (WPI-MANA), National Institute for Material Sciences (NIMS), 1-1 Namiki, Tsukuba, Ibaraki 305-0044, Japan

$\S$ Institute of Chemical Research of Catalonia (ICIQ), Barcelona Institute of Science and Technology (BIST), 43007 Tarragona, Spain; Department de Química Analítica i Química Orgànica, Universitat Rovira i Virgili, 43007 Tarragona, Spain

\section{AUTHOR INFORMATION}

\section{Corresponding Author}

We-Hyo Soe - Centre d'Elaboration de Matériaux et d'Études Structurales (CEMES), Centre National de la Recherche Scientifique (CNRS), 29 Rue J. Marvig, BP 94347, 31055 Toulouse Cedex, France; Email : we-hyo.soe@cemes.fr 
ABSTRACT: A specifically designed aza-starphene molecule is presented where contacting one, two and/or three single $\mathrm{Al}$ ad-atoms allows this molecule to function as a "3-inputs \& 2-outputs" digital full adder on a $\mathrm{Au}(111)$ surface. Sequentially positioning single $\mathrm{Al}$ ad-atoms, with an atomic precision, to interact with aza-starphene inputs one classical digit per $\mathrm{Al}$, which is converted to quantum information by the molecule. The intramolecular logical calculations do not require a solid-state digital full adder cascaded like architecture. The measured Boolean truth table results in part from the quantum level repulsion effect and in part from a non-linear magnetic effect also intrinsic to the aza-starphene molecule with its contacted $\mathrm{Al}$ ad-atoms.

\section{TOC GRAPHICS}

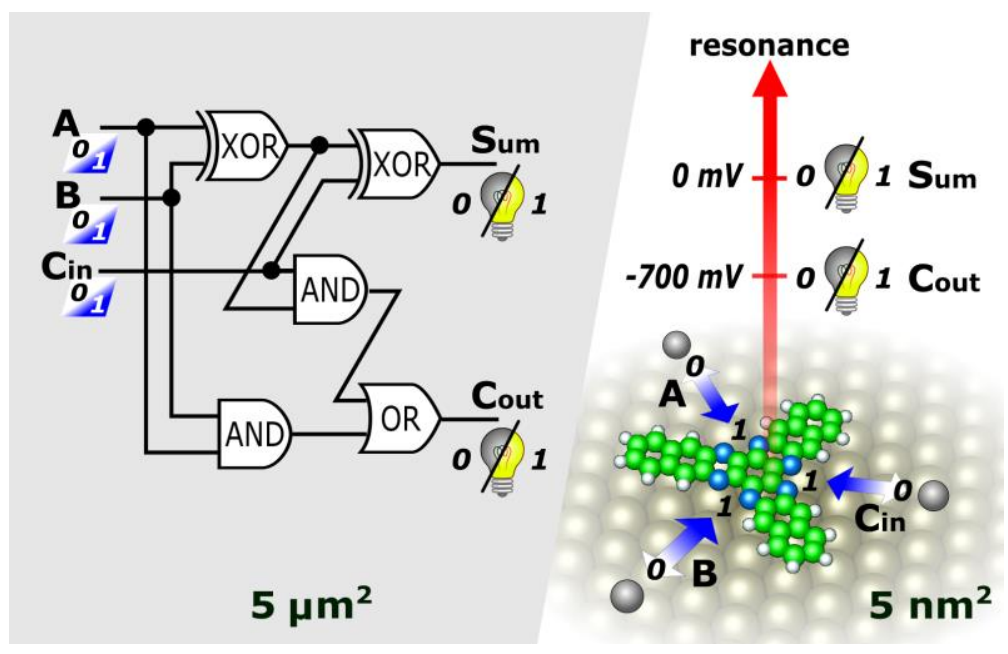

KEYWORDS single molecule 1-bit full adder, ground state manipulation, Kondo state, LT-STM, scanning tunneling spectroscopy 
At low temperature, single atom manipulations using a scanning tunneling microscope (LTUHV-STM) can be used to enter analogue ${ }^{1}$ or digital ${ }^{2}$ classical information inside a single molecule. The quantum intrinsic response of the molecule to those classical inputs is normally a shift in energy of its ground and first electronic excited states according to the required logical truth table. NOR, NAND and XOR 2-inputs, 1-output elementary logic gates have been designed and studied experimentally this way, ${ }^{2-4}$ following the quantum Hamiltonian computer (QHC) approach. ${ }^{5}$ It had also been demonstrated theoretically that the QHC approach to quantum computation can reach at least the complexity of a $2 \times 2$ words digital adder without the need of cascading elementary QHC gates. $^{6}$ As presented in Fig. 1 and inside a QHC molecule, the calculation scheme presented here for a 3-inputs \& 2-outputs digital full adder is not demanding any cascading of AND, XOR and OR two inputs logic gates as it is usually the case for the design of electronic digital adders. ${ }^{7}$

In our experimental approach to $\mathrm{QHC}$, manipulating one single metal ad-atom in electronic contact with the molecule is a classical action: a " 0 " logical binary input when there is no and a "1" logical binary input when there are electronic interactions between the molecule ant the adatom. This classical one-bit information is transduced by the electronic quantum states of the molecule and is equivalent to load one-bit of quantum information inside this molecule. After this transduction, the intramolecular Boolean calculations is performed. For reading the logical outputs, we measure the tunnel electronic resonance spectrum of the single molecule while inserted in a tunneling junction. ${ }^{2-4}$ For a $\mathrm{QHC}$ molecule logic gate with more than one logical output, two strategies are possible for reading the logical outputs: recording those resonances (1) at the same energy but at different locations along the molecule or (2) at the same location but at different 
energies. ${ }^{6,8}$ For the two logical outputs digital full adder of the present study, we have followed strategy (2) as presented in Fig. 1.

We have designed the large new aza-starphene molecule (Fig. 2(a)) by introducing 3 pyrazine molecular groups in the central node of a long starphene. ${ }^{9}$ For the metal inputs, we have selected single $\mathrm{Al}$ ad-atom since the oxidation state of a single $\mathrm{Al}$ on $\mathrm{Au}(111)$ is between $\mathrm{Al}(\mathrm{I})$ and $\mathrm{Al}(\mathrm{II}){ }^{10}$ The LT-UHV-STM molecular manipulations are used to stabilize a single Al ad-atom in the middle of two nitrogen atoms on different branches of an aza-starphene molecule and leads to a change of its tunneling spectrum.

To benefit from the complete calculating power of our aza-starphene molecule, we have also exploited the magnetic properties of aza conjugated molecules when coordinated with $\mathrm{Al}$ ad-atoms on the $\mathrm{Au}(111)$ surface. ${ }^{10}$ As described below, it brings the sum logical output of our aza-starphene molecule-adder exactly at a $\mathrm{V}=0$ bias voltage according to Fig $1 \mathrm{~b}$. It can be detected by a Kondo resonance because this molecule is demonstrating a local magnetic moment with one and three coordinated $\mathrm{Al}$ ad-atoms on the molecule physisorbed on the $\mathrm{Au}(111)$ surface.

The experimental results presented here were obtained with a low temperature ultra-high vacuum 4-STM instrument (LT-UHV 4-STM) having four independent LT-UHV-STM able on the same surface to operate in parallel with the same $\mathrm{d} / \mathrm{dV}$ spectral and atomic manipulation precisions than a single tip LT-UHV-STM. ${ }^{11}$ The atomically clean $\mathrm{Au}(111)$ single crystal surface was prepared by a repeated Ar+ sputtering and heating up to $780 \mathrm{~K}$ in $\mathrm{UHV}$. The $\mathrm{Au}(111)$ surface was exposed to a low flux of aza-starphene molecules to obtain a very low sub-monolayer coverage 
at room temperature. This sample was then transferred to the LT-UHV 4-STM cold sample stage maintained at $4.7 \mathrm{~K}$. Then, a very small amount of $\mathrm{Al}$ atoms was evaporated on this cold $\mathrm{Au}(111)$ surface through a small aperture equipped with a copper shutter through the liquid nitrogen shield of our LT-UHV 4-STM cryostat. Tungsten STM tips were electrochemically etched. STM images were recorded indistinctively on any of the four STM of our LT-UHV 4-STM using a nominal 500 $\mathrm{mV}$ bias voltage and a $100 \mathrm{pA}$ feedback loop set up current. After those 2 successive depositions, a typical LT-UHV STM image of the Au(111) surface is presented in Fig. 2(b). The Au(111) herringbone characteristic surface reconstruction is well imaged together with the aza-starphene molecule and the $\mathrm{Al}$ ad-atoms.

Contacting the aza-starphene with one, two and three Al ad-atoms was performed by STM manipulating this molecule towards the $\mathrm{Al}$ and then recording successively the $\mathrm{dI} / \mathrm{dV}$ electronic tunneling spectra for one, two and three $\mathrm{Al}$ ad-atoms coordinated to the molecule. We can go backward in this logical input sequence by manipulating again the molecule away from the $\mathrm{Al}$ adatoms. An aza-starphene molecule was STM manipulated using a $50 \mathrm{M} \Omega$ tunneling junction resistance, and it reduced down to $12 \mathrm{M} \Omega$ with the increase of the number of $\mathrm{Al}$ atoms coordinated to the molecule. The dI/dV differential conductance tunneling spectra of the " STM tip - molecule - Au(111) surface" tunnel junction were recorded using a lock-in amplifier technique with $8 \mathrm{mV}$ bias voltage swiping amplitude. While ramping up and down the bias voltage on this junction, each recorded maximum defines a tunnel electronic resonance corresponding to the opening of an elastic or inelastic tunneling channel through the junction. ${ }^{1,2}$ 
The native $\mathrm{dI} / \mathrm{dV}$ spectrum of a single isolated aza-starphene molecule adsorbed on $\mathrm{Au}(111)$ is presented in Fig. 3 together with its so-called molecular orbital mapping. In the $[-2.0 \mathrm{~V},+2.0 \mathrm{~V}]$ voltage range, three electronic tunneling resonances were observed. They respectively correspond to the $\mid \mathrm{S} 0>$ ground, $\mid \mathrm{S} 1>$ and $\mid \mathrm{S} 2>$ excited state electronic resonances of the aza-starphene molecule. They are accessible via the millions of elementary hole and electron transfer processes through the molecule giving rise to the tunneling current: the $\mid \mathrm{S} 0+>$ virtual state for $\mid \mathrm{S} 0>$ and the $\mid \mathrm{S} 1->$ and $\mid \mathrm{S} 2->$ for the $\mid \mathrm{S} 1>$ and $\mid \mathrm{S} 2>$ states respectively. At constant current, the dI/dV mapping is capturing the main mono-electronic component of those states i.e. the one with the largest weight in the electronic state decomposition in Slater determinants and the largest effective electronic coupling between the tip end atom apex and the surface through the molecule. When scanning the tip apex over the molecule at constant current intensity, it provides an HOMO map of $\mid \mathrm{S} 0>$, an LUMO of $\mid \mathrm{S} 1>$ and approximatively the LUMO+1 map of $\mid \mathrm{S} 2>$. For this last one and when reaching the $\mid \mathrm{S} 2>$ resonance, $\mid \mathrm{S} 1>$ also contributes because of its tunneling resonance overlapping with the $\mid \mathrm{S} 2>$ resonance.

For a two logical outputs QHC molecule logic gate and as describe in the introduction, there are two distinct output strategies: tracking the tunneling resonances at the same energy but at different locations along the molecule or at the same location on the molecule but at different energies. ${ }^{6}$ As presented in Fig. 4 and after a careful mapping of the aza-starphene-(Al)n complex for $n=0,1,2$ and 3 , there is a node of the HOMO at the center of the molecule for $\mathrm{n}=0$ and 1 and a maximum for $\mathrm{n}=2$ and 3 . This is exactly what is expected for the carry logical output of a digital full adder. The sum logical output is captured at the center of the molecular complex for $n=1$ and 3 via the recording of a Kondo resonance. This Kondo resonance is a $\mathrm{dI} / \mathrm{dV}$ spectral signature indicating 
that the aza-starphene-(Al)n complexes are magnetic for $\mathrm{n}=1$ and 3 . A complete analysis of the magnetic properties of those 2 complexes is now further explored, in particular the value of the corresponding magnetic moment. ${ }^{12}$ The Fig. $4 \mathrm{~d} / \mathrm{dV}$ resonance location and their companion molecular orbital mapping is demonstrating how the electronic states of the aza-starphene-(Al)n complexes are shifted as a function of the number of coordinated $\mathrm{Al}$ ad-atoms. For $\mathrm{n}=1$, the complex is magnetic, ${ }^{12}$ and $\mid \mathrm{S} 0>$ and $\mid \mathrm{S} 1>$ are moderately shift up and down in energy respectively. For $\mathrm{n}=2$, the shift up and down are more than $+1 \mathrm{eV}$ for $\mid \mathrm{S} 0>$ and $-0.5 \mathrm{eV}$ for $\mid \mathrm{S} 1>$. For $\mathrm{n}=3$, the complex is magnetic again with certainly a large magnetic moment. ${ }^{12}$ Its $\mid \mathrm{S} 0>$ is pushed down but we do not succeed to capture the $\mid \mathrm{S} 1>$ resonance in the stability voltage range of our STM experiments.

The logical operation of the aza-starphene molecule is analyzed in Fig. 5 and compared with the expected full adder Boolean truth table. The Fig. $5 \mathrm{dI} / \mathrm{dV}$ spectra were recorded with the STM tip end atom apex positioned on the central aza-starphene phenyl group where the logical outputs are recorded symmetrically relative to the logical inputs. In Fig. 5, the raw $A u(111)$ and noncoordinated aza-starphene $\mathrm{dI} / \mathrm{dV}$ spectra are included as a reference. They illustrate the $\mathrm{Au}(111)$ surface state at $-500 \mathrm{mV}$ and the absence of tunneling electronic resonances coming from the azastarphene in the $[-1 \mathrm{~V},+1 \mathrm{~V}]$ voltage range. The logic output sum resonance is captured at $0.0 \mathrm{~V}$ and the carry resonance at $-0.7 \mathrm{~V}$. The complete truth table of a digital full adder is verified by our aza-starphene molecule.

From the logical input to the readout, our molecule logic gate operation is very slow because of the lengthy STM single atom and molecule manipulation protocols used. Once one $\mathrm{Al}$ ad-atom 
coordinates to the two nitrogen on different pyrazines, the intramolecular spectral response time of the molecule will be quite fast, about a femtosecond for such a complex electronic cloud (to be compared to $10^{-16} \mathrm{~s}$ to stabilize the electronic cloud of a single atom ${ }^{13}$ ). It is the time interval for the aza-starphene-Al complex electronic levels to be stabilized again for example from the native tunneling resonance distribution of the $(0,0,0)$ logical input configuration to one of the new spectra recorded for the other logical configurations. In the future, single molecular switches will certainly be incorporated to the molecular design to speed up the logical input for example by using light. ${ }^{14}$ A silicon based 3-inputs 2-outputs Boolean full adder is actually occupying a $50 \mu \mathrm{m}^{2}$ surface area corresponding to the state-of-the-art micro-electronics industry while the same function only needs $5 \mathrm{~nm}^{2}$ within our single molecule i.e. seven orders of magnitude difference. We benefit here from the fact that a QHC design does not require a cascade of elementary to elementary logic gates, the Boolean function resulting from a quantum control in the quantum state space. Finally, our strategy to calculate in the quantum state space playing with the quantum level repulsion effect can be applied on different atomic scale supports like graphene ${ }^{15}$ or $\mathrm{Si}(100) \mathrm{H}^{16}$ surfaces. The presented full adder is an experimental proof of concept that the QHC approach can go over the elementary "2-inputs, 1-output" logic function with an exceptional size reduction of the amount of materials required to perform a complex logic function. By using single atom manipulations, the construction aza-starphene-(Al)n complexes while two of them being structurally asymmetric is also a nice example of the recent concept of coordination asymmetry. It will certainly help for the design of more complex Boolean logic functions embedded in a single molecule.

\section{Notes}

The authors declare no competing financial interest. 


\section{ACKNOWLEDGMENT}

This work has been supported by the European Union Horizon 2020 FET open project "Mechanics with Molecule(s)" (MEMO, grant 766864) and the MANA-NIMS MEXT WPI program.

\section{REFERENCES}

(1) Manzano, C.; Soe, W.-H.; Grisolia, M.; Hliwa, M.; Joachim, C. Manipulation of a Single Molecule Ground State by means of Gold Atom Contacts, Chem. Phys. Lett. 2013, 587, 35-39, DOI:10.1016/j.cplett.2013.09.039. 
(2) Soe, W.-H.; Manzano, C.; Renaud, N.; de Mendoza, P.; De Sarkar, A.; Ample, F.; Hliwa, M.

Echevaren, A. M.; Chandrasekhar, N.; Joachim, C. Manipulating Molecular Quantum States with Classical Metal Atom Inputs: Demonstration of a Single Molecule NOR Logic Gate, ACS Nano 2011, 5 ,1436-1440, DOI:10.1021/nn1033899.

(3) Skidin, D.; Faizy, O.; Krüger, J.; Eisenhut, F.; Jancarik, A.; Nguyen, K.-H.; Cuniberti, G.; Gourdon, A.; Moresco, F.; Joachim, C. Unimolecular Logic Gate with Classical Input by Single Gold Atoms, ACS Nano 2018, 12, 1139-1145, DOI:10.1021/acsnano.7b06650.

(4) Soe, W.-H.; Manzano, C.; Joachim, C. A Tetrabenzophenazine Low Voltage Single Molecule XOR Quantum Hamiltonian Logic Gate, Chem. Phys. Lett. 2020, 748, 137388, DOI:10.1016/j.cplett.2020.137388.

(5) Renaud, N.; Joachim, C. The Design and Stability of NOR and NAND Logic Gates Constructed with Only 3 Quantum States, Phys. Rev. A 2008, 78, 062316, DOI:10.1103/PhysRevA.78.062316.

(6) Namarvar, O. F.; Giraud, O.; Georgeot, B.; Joachim, C. Quantum Hamiltonian Computing Protocols for Molecular Electronics Boolean Logic Gates, Quantum Sci. Technol. 2019, 4, 035009, DOI:10.1088/2058-9565/ab2412.

(7) Moris Mano, M. Digital Logic and Computer Design; Prentice-Hall, 1979.

(8) Jensen, P. W. K.; Jin, C.; Dallaire-Demers, P.; Aspuru-Guzik, A.; Solomon, G. C. Molecular Realization of a Quantum NAND Tree, Quantum Sci. Technol. 2019, 4, 015013, DOI:10.1088/2058-9565/aaf24b. 
(9) Soe, W.-H.; Manzano, C.; de Mendoza, P.; McGonigal, P. R.; Echavarren, A. M.; Joachim, C. Long Starphene Single Molecule NOR Boolean Logic Gate, Surf. Sci. 2018, 678, 163-168, DOI:10.1016/j.susc.2018.04.020.

(10) Soe, W.-H.; Manzano, C.; Robles, R.; Lorente, N.; Joachim, C. On-Surface Atom-by-AtomAssembled Aluminum Binuclear Tetrabenzophenazine Organometallic Magnetic Complex, Nano lett. 2020, 20, 384-388, DOI:10.1021/acs.nanolett.9b04040.

(11) Yang, J.; Sordes, D.; Kolmer, M.; Martrou, D.; Joachim, C. Imaging, Single Atom Contact and Single Atom Manipulation at Low Temperature using the New ScientaOmicron LT-UHV 4 STM, Eur. Phys. J. Appl. Phys. 2016, 73, 10702, DOI:10.1051/epjap/2015150489.

(12) Soe, W.-H.; Robles, R.; de Mendoza, P.; Echavarren, A. M.; Lorente, N.; Joachim, C. Doublet-Singlet-Doublet Transition in a Single Organic Molecule Magnet On-Surface Constructed with up to 3 Aluminum Atoms, Nano lett. 2021 (submitted).

(13) Kienberger, R.; Goulielmakis, E.; Uiberacker, M.; Baltuska, A.; Yakovlev, V.; Bammer, F.; Scrinzi, A.; Westerwalbesloh, Th.; Kleineberg, U.; Heinzmann, U.; Drescher, M.; Krausz, F. Atomic Transient Recorder, Nature 2004, 427, 817-821, DOI:10.1038/nature02277.

(14) Wen, Y.; Yi, W.; Meng, L.; Feng, M.; Jiang, G.; Yuan, W.; Zhang, Y.; Gao, H.; Jiang, L.; Song, Y. Photochemical-Controlled Switching Based on Azobenzene Monolayer Modified Silicon (111) Surface, J. Phys. Chem. B 2005, 109, 30, 14465-14468, DOI:10.1021/jp044256t.

(15) Srivastava, S.; Kino, H.; Joachim, C. Quantum Half-Adder Boolean Logic Gate with a Nano-Graphene Molecule and Graphene Nano-Electrodes, Chem. Phys. Lett. 2017, 667, 301-306, DOI:10.1016/j.cplett.2016.11.009. 
(16) Kolmer, M.; Zuzak, R.; Dridi, G.; Goldewski, S.; Joachim, C.; Szymonski, M. Realization of a Quantum Hamiltonian Boolean Logic Gate on the Si(001):H Surface, Nanoscale 2015, 7, 12325-12330, DOI:10.1039/c5nr01912e. 
(a)

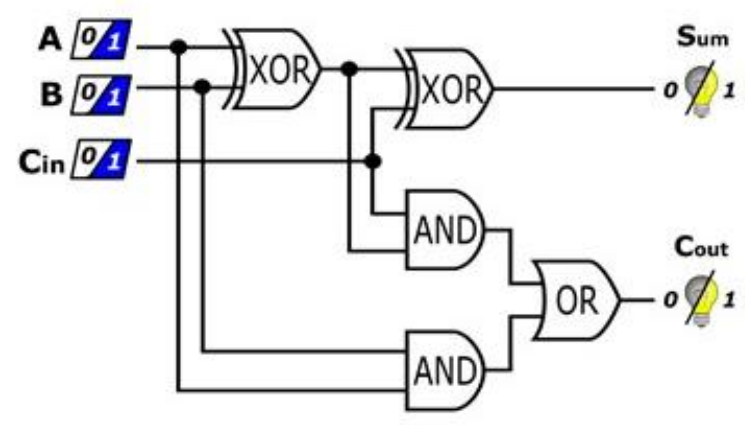

(b)

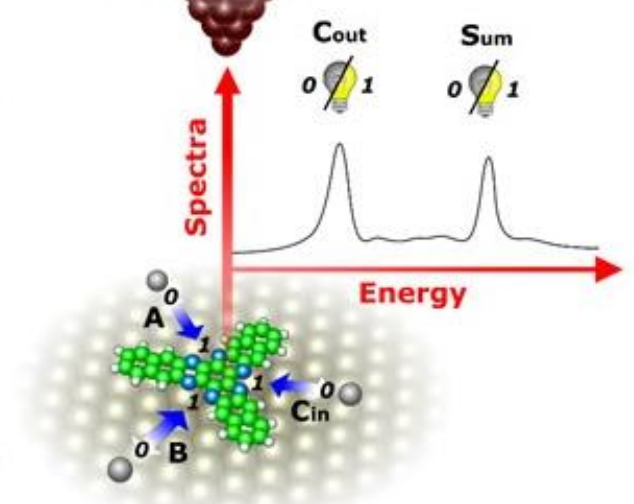

Figure 1. For a 3-inputs \& 2-outputs" digital full adder, the difference between (a) a standard cascaded like electronic circuit with the A, B \& Cin (Carry in) logical inputs, the elementary XOR, AND \& OR logic gate, and the Sum \& Cout (Carry out) outputs involving many electrical wiring and interconnects and (b) the studied aza-starphene QHC molecule where the chemical structure of the molecule is not showing any cascading of elementary logic gates nor wiring or intramolecular interconnects. On the aza-starphene molecule, the A, B \& Cin logical inputs are the $3 \mathrm{Al}$ ad-atoms and the Sum \& Cout outputs are spectral outputs, positioning always the STM tip apex at the center of the aza-starphene molecule and recording the corresponding $\mathrm{dI} / \mathrm{dV}$ tunnel spectra for reading the outputs. For this aza-starphene QHC molecule, the Sum output is positioned at a zero bias voltage and the Cout at $-0.700 \mathrm{~V}$ below the metal supporting surface Fermi level. With such a recording, the saturation of the quantum repulsion effect ${ }^{15}$ used for the QHC design is avoided for the sum (see Fig. 5 for the corresponding truth table). 
(a)

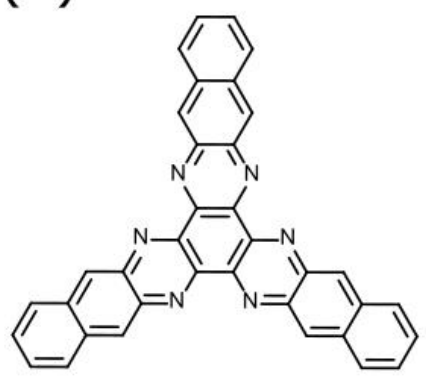

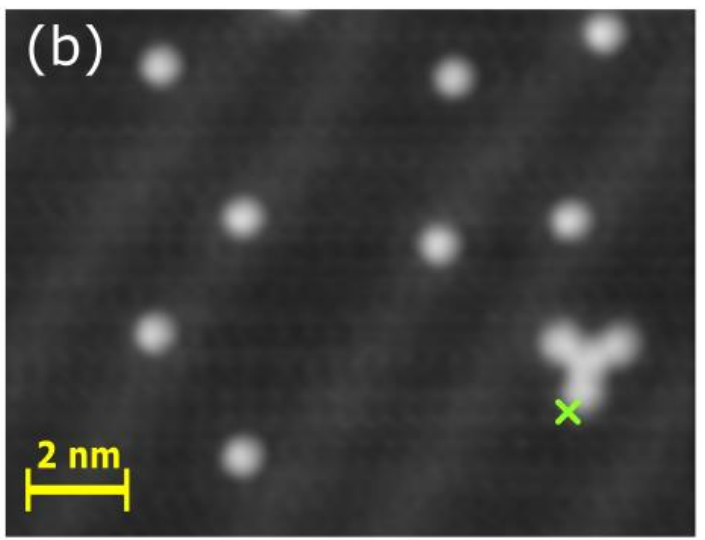

Figure 2. (a) The chemical structure of the aza-starphene molecule and (b) a large scan constant current STM image recorded using one tip of the LT-UHV 4-STM showing the Au(111) reconstructions, one aza-starphene molecule and $8 \mathrm{Al}$ ad-atoms. Image conditions: $100 \mathrm{pA}$ and $500 \mathrm{mV}$. Herringbone corrugation is $\Delta \mathrm{z}=30 \mathrm{pm}$. The green cross is indicating the STM tip apex position for recording the Fig. $3 \mathrm{dI} / \mathrm{dV}$ spectrum. 

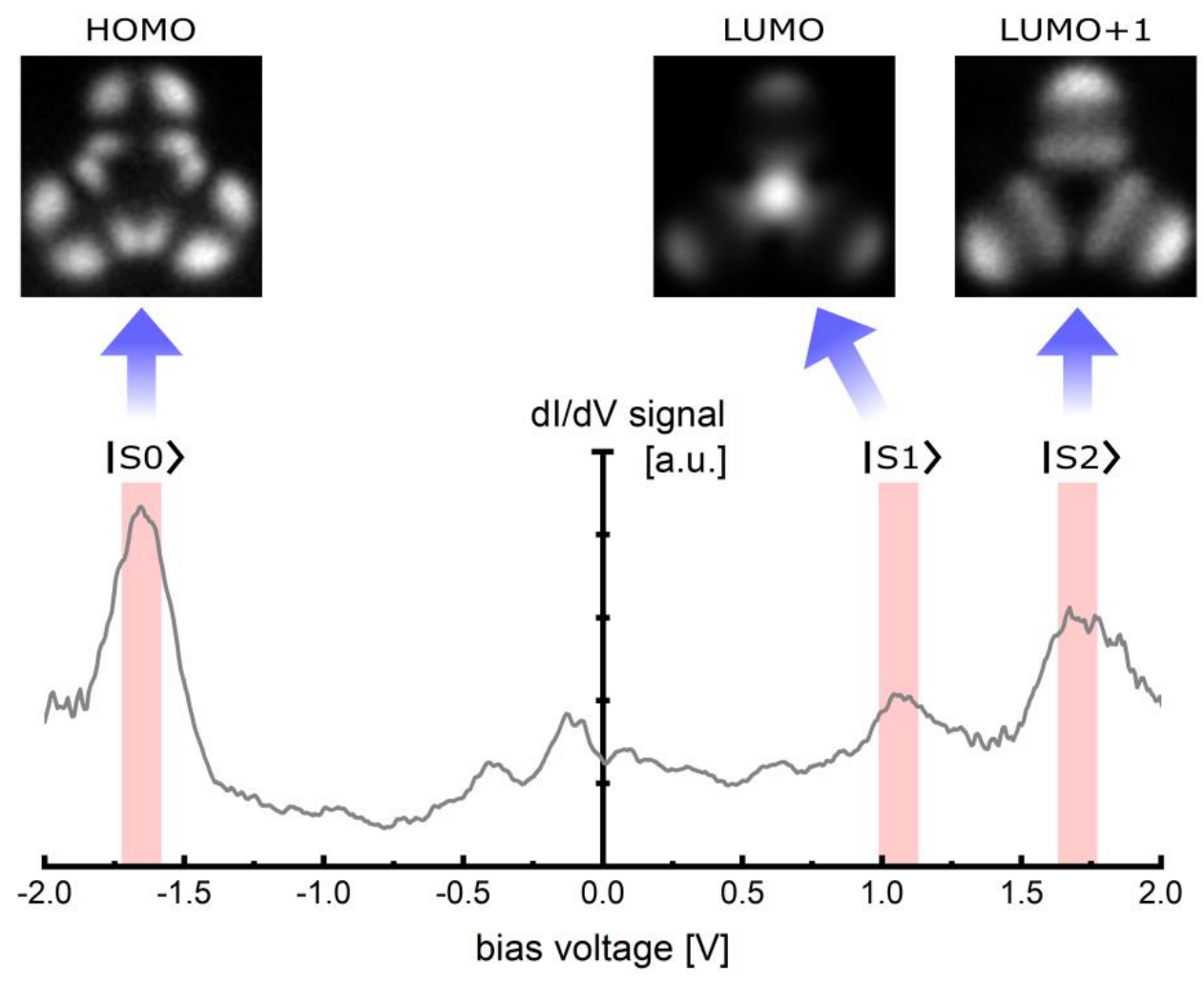

Figure 3. The native dI/dV spectrum of a free aza-starphene with its $|\mathrm{S} 0\rangle,|\mathrm{S} 1\rangle$ and $|\mathrm{S} 2\rangle$ tunneling resonances. Their corresponding constant current $\mathrm{d} / / \mathrm{dV}$ mapping is given and was recorded exactly at each resonance maximum. The image contrast is corresponding to the maximum monoelectronic contribution to the $|\mathrm{S} 0\rangle,|\mathrm{S} 1\rangle$ and $|\mathrm{S} 2\rangle$ complete electronic structure showing respectively the HOMO, LUMO and LUMO+1 tunneling contrast. ${ }^{2}$ Tip position for this spectrum is indicated on Fig. 1(b) by a green cross. 

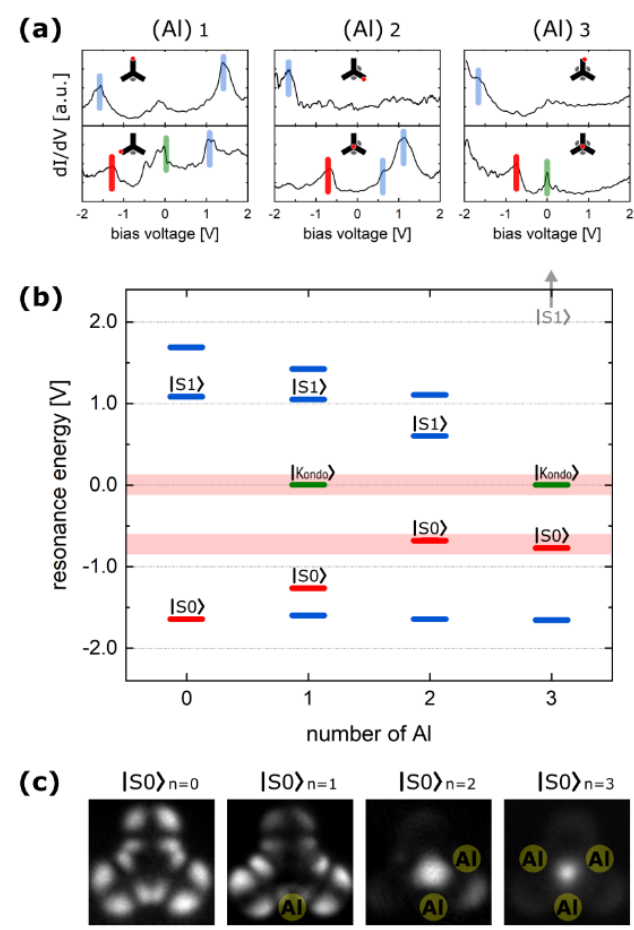

Figure 4. (a) The selected $[-2 \mathrm{~V},+2 \mathrm{~V}] \mathrm{dI} / \mathrm{dV}$ spectra to locate the tunneling resonance of the azastarphene-(Al)n complexes as a function of the number $\mathrm{n}=1$ to 3 of $\mathrm{Al}$ coordinated ad-atom including in green (also in b) the Kondo resonance signature of a magnetic state of the complexes for $n=1$ and $n=3$. The difference between two spectra of each complex is the tip location (which is indicated by red dot on the simplified molecular diagram for each spectrum in (a)) during spectroscopic measurement. The shape of resonance peaks and their intensities are related to where the tip end apex state couples locally with each molecular electronic state having its own molecular orbital (MO) content distribution in real space. (c) The corresponding $\mid \mathrm{S} 0>$ experimental HOMO molecular orbital mapping showing a central node for $\mathrm{n}=0$ and 1 , and a central MO maximum for $\mathrm{n}=2$ and 3 . For the logical output reading in (b), the $0.0 \mathrm{~V}$ pink area is for the digital sum and the $-0.7 \mathrm{~V}$ pink area is for the digital carry (see Fig. 5 for the corresponding logical truth table). Notice that the $\mid \mathrm{S} 1>$ resonance for $\mathrm{n}=3$ is out of the range of the $\mathrm{dI} / \mathrm{dV}$ STS experiment (grey arrow up, 4th spectra column on the right in (b)). 


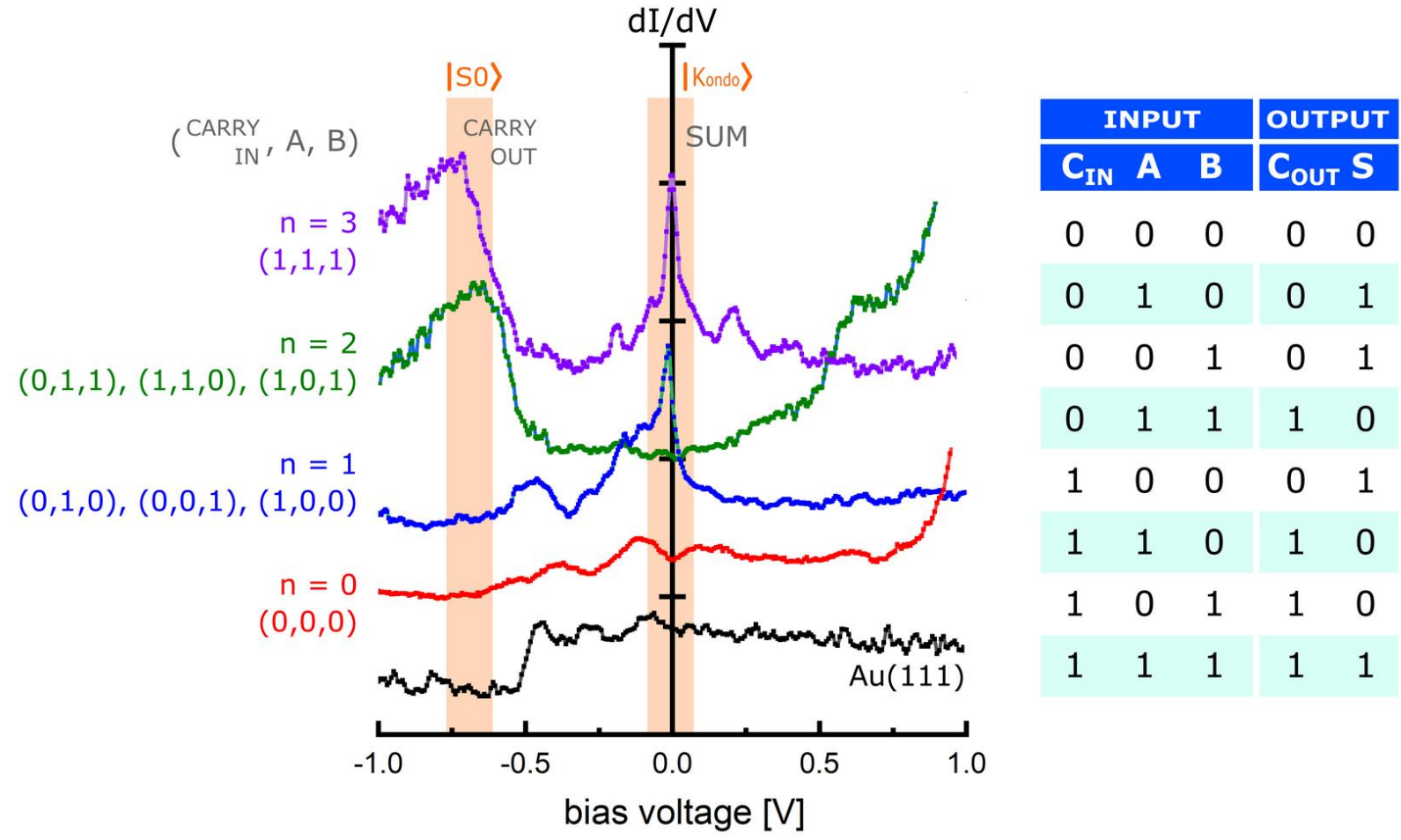

Figure 5. The detailed $[-1 \mathrm{~V},+1 \mathrm{~V}] \mathrm{dI} / \mathrm{dV}$ spectra from $\mathrm{n}=0$ to $\mathrm{n}=3$ coordinated Al-atom to the aza-starphene molecule with a specific recording of the Kondo resonance for $n=1$ and $n=3$. To record those $\mathrm{dI} / \mathrm{dV}$ spectra, the tip apex was located on the central aza-starphene molecule where there is a maximum of conductance as presented in Fig. 4. The Boolean truth table of a full binary adder is also recalled. The resonance existing at $-0.7 \mathrm{~V}$ or at $0.0 \mathrm{~V}$ bias voltage is encoding for a logical "1" for the Carry-out and for the Sum-out respectively. 